\title{
Sufrimiento psicológico y malestar emocional en las personas migrantes sin hogar.
}

Psychological and emocional distress in immigrant homeless.

\author{
Miguel Angel Navarro-Lashayas a . \\ ${ }^{a}$ Psicólogo. Doctor en Migraciones Internacionales Contemporáneas. Universidad Pontificia de Comillas. \\ Ekimen Elkartea. OME-AEN. Portugalete, Bizkaia, España.
}

Correspondencia: Miguel Angel Navarro-Lashayas (m.lashayas@gmail.com)

Recibido: 16/12/2013; aceptado: 26/01/2014

RESUMEN: La mayoría de la investigación en torno a la exclusión social se ha centrado en psicopatología y poco se han estudiado otras variables. El artículo muestra las relaciones entre el sufrimiento psicológico y malestar emocional con variables sociodemográficas, estrés aculturativo, apoyo social, abusos de sustancias, sucesos vitales estresantes y cambios en la identidad en una muestra de 107 personas migrantes sin hogar. Para ello se han realizado entrevistas en profundidad y autoinformes analizando las relaciones entre las variables mediante diversas técnicas de análisis de datos. Los resultados muestran la importancia de los recursos materiales en el bienestar psicológico, así como la alta influencia de los sucesos vitales estresantes sufridos por la persona, el apoyo social recibido y otras variables vinculadas con la experiencia de exclusión social como la deshumanización, la falta de control sobre la propia vida y los cambios identitarios. Todo ello nos debe llevar a reflexionar sobre la manera que desde la psicología y psiquiatrías nos acercamos al estudio de los problemas sociales, dando un excesivo énfasis al diagnóstico psicopatológico en perjuicio de otras variables importantes como las citadas.

PALABRAS CLAVE: Problemas Sociales, Marginación Social, Migración Internacional, Impacto Psicosocial, Estrés Psicológico.
ABSTRACT: Most research on social exclusion has focused on psychopathology and some other variables had been little studied. The article explores the relationship between psychological and emotional distress with sociodemographic variables, acculturative stress, social support, substance abuse, stressful life events and changes of identity in a sample of 107 homeless migrants. This has been made interviews and self-reports exploring the relationships between variables using various techniques of data analysis. The results show the importance of material resources in psychological wellbeing, and the high influence of stressful life events experienced by the person, the social support, and other variables related to the experience of social exclusion such as dehumanization, lack of control over their own lives and changes in the identity. All of which leads us to reflect on how psychology and psychiatry approaches the study of social problems, giving too much emphasis to psychopathologic diagnoses, detriment of other important variables.

KEY WORDS: Social Problems, Social Marginalization, Emigration and Immigration, Psychosocial Impact, Stress, Psychological.

Agradecimientos:

A las entidades sociales de la plataforma BesteBi por su trabajo diario a favor de las personas sin hogar y por facilitarme los contactos para realizar las entrevistas. A todas las personas migrantes sin hogar por su apertura y ganas de compartir conmigo su experiencia. 


\section{Introducción.}

Mucho se ha escrito en torno a las personas sin hogar y la salud mental. Uno de los temas que más investigación ha generado es el papel que juega el problema de salud mental y de abuso de sustancias en la etiología y mantenimiento de la situación sin hogar. La calle puede ser el destino de personas con problemas de salud mental o abuso de sustancias que no cuentan con una red social de apoyo lo suficientemente sólida (fundamentalmente la familia), y donde ha fallado también las redes institucionales encargadas de proveer apoyo (fundamentalmente red sanitaria y de protección social). Por otra parte, algunos autores sostienen que la exclusión extrema que sufren las personas que viven en la calle produce unos efectos psicológicos que precipitan la aparición de problemas de abuso de sustancias y de salud mental (1-2).

Los datos más sólidos acerca de la relación entre salud mental y personas sin hogar a nivel internacional, sitúan la prevalencia de los trastornos graves entre el 25\% y el 35\% (3). En un meta análisis realizado recientemente (4), con los resultados de 29 trabajos y una muestra conjunta de 5.684 personas sin hogar, se muestra una gran heterogeneidad en los resultados, con tasas de dependencia del alcohol entre el 8,5\% y el 58,1\% (prevalencia media 37,9\%), dependencia de otras sustancias entre el 4,7\% y el 54,2\% (prevalencia media 24,4\%), trastornos psicóticos entre el 2,8\% y el 42,3\% (prevalencia media 12,7\%), depresión mayor con una prevalencia media de $11,4 \%$ y trastornos de personalidad con una prevalencia media de $23,1 \%$.

Las cifras de personas sin hogar en España con trastornos psicológicos o psiquiátricos importantes se sitúan entre el 20\% y el 52\% (5-8). La divergencia de cifras es explicada por los diferentes instrumentos utilizados para detectar trastornos. Así, en una investigación donde se recogen datos referentes a 23 investigaciones realizadas en España entre 1975 y 2003 (9), los datos se igualan cuando los estudios utilizan los mismos instrumentos estandarizados de diagnóstico. En dos estudios en los que se utiliza el Composite Diagnostic International Interview (CIDI) la tasa de esquizofrenia se sitúa entre el 4\% y el 12\%, la de depresión mayor entre el $15 \%$ y el $20 \%$, la dependencia o abuso de alcohol entre el $21 \%$ y $26 \%$ y la de drogas entre el $12 \%$ y el $13 \%$.

En cualquier caso, comparando estas cifras con las que arrojan los estudios para población general, no cabe la menor duda de que nos encontramos ante una problemática importante. El estudio más reciente sobre epidemiología de los trastornos psiquiátricos en la población general en España (European Study of the Epidemiology of Mental Disorders -ESEMED-), estima en un 8,4\% la población general con un trastorno mental en los últimos 12 meses. El estudio también utiliza el CIDI como herramienta de diagnóstico y cifra en un 3,9\% la tasa de prevalenciaaño y $10,5 \%$ prevalencia-vida el trastorno de depresión mayor, y en un $0,7 \%$ la tasa 
de esquizofrenia (10), lo cual supone que los datos de las personas sin hogar cuadriplican los de la población general en diagnósticos como el de la esquizofrenia.

Las personas migrantes también han sido objeto de investigación desde la psicología y la psiquiatría. Sobre las posibilidades de que la migración pueda ser el desencadenante de enfermedades mentales hay posturas enfrentadas. A pesar de que dicha asociación se ha dado por supuesta, cada vez son más los investigadores que afirman que la emigración per se, no produciría un incremento en el riesgo de padecer enfermedades mentales. Ejemplo de ello es un meta análisis de la literatura internacional que muestra que no existen evidencias concluyentes de mayor riesgo de trastornos de ánimo asociados a la migración (11).

Los enfoques actuales ponen el énfasis en la situación social que vive la persona y en el estrés como posibles desencadenantes de problemáticas de salud mental. Ejemplo de ello es el Síndrome de Ulises (síndrome del inmigrante con estrés crónico y múltiple), (12-13) que relaciona el estrés con las condiciones de vida en las que se tienen que desenvolver las personas (soledad, fracaso migratorio, lucha por la supervivencia y miedo), siendo potenciados los estresores por su multiplicidad, cronicidad, intensidad, ausencia de sensación de control y ausencia de red de apoyo social. Otros autores (14), han planteado un modelo de estrés de la migración basándose en diferentes componentes como la nostalgia, el duelo que implica dejar atrás determinado modo de vida, el choque cultural o proceso aculturativo, la frecuente experiencia de discriminación y las condiciones de vida. Aunque conceptualmente se compone de diferentes factores, desde el punto de vista psicométrico el estrés de la inmigración es unitario. La inmigración es estresante en la medida que es sentida y vivida como tal por la persona.

La mayoría de estudios realizados en España con ambas poblaciones se han centrado en la psicopatología y apenas han prestado atención a variables como el malestar psicológico o el sufrimiento emocional, dejando de lado las vivencias personales de aquellos que sufren exclusión social o son víctimas de la discriminación. La investigación que se presenta se centra en dichas vivencias y su repercusión para la persona.

\section{Método.}

Se han realizado un total de 107 entrevistas a personas migrantes sin hogar, siguiendo como criterio de estratificación el alojamiento actual. Las dos sub muestras responden a las siguientes características:

- Una muestra de 60 personas que viven en la calle, estructuras temporales, edificios abandonados, etc. o bien combinando dichas situaciones con estancias cortas (menos de una semana) en recursos de alojamiento de emergencias. 
- Una muestra de 47 personas que residen en alojamientos para personas sin hogar de media-larga estancia (tres o más meses de estancia) que han tenido historia reciente (menos de un año) de paso por las situaciones anteriormente descritas.

Las personas fueron contactadas gracias a la labor de 16 recursos para personas sin hogar e inmigrantes de la ciudad de Bilbao. Las entrevistas fueron realizadas en los locales de las asociaciones, albergues, comedores sociales y centros de día.

Las variables utilizadas son:

Variables sociodemográficas y de calle:

- Información sociodemográfica: Sexo, edad, país de nacimiento y nivel de estudios.

- Proyecto migratorio: Tiempo de estancia en España, tiempo de estancia en Bilbao, recorrido por otras provincias.

- Documentación: Situación documental, razón de no tener "papeles", empadronamiento, lugar y duración del empadronamiento, razones de no estar empadronado.

- Trabajo e ingresos económicos: Prestaciones sociales, trabajos que realiza, tiempo trabajado.

- Información de calle: Tiempo de estancia en la calle, tipos de alojamientos en los que ha estado.

Estrés aculturativo: Se ha utilizado la escala Barcelona de estrés del inmigrante (BISS) (14) con 42 ítems y un formato de respuesta tipo Likert de 1 (totalmente en desacuerdo) a 4 (totalmente de acuerdo). La escala está compuesta por 4 factores: Discriminación percibida, estrés o tensión del contacto intercultural, nostalgia y estrés psicosocial general.

Apoyo social: Se ha utilizado una escala construida ad hoc basada en la versión adaptada a la población de inmigrantes africanos (15) de la entrevista ASSIS (16) para la parte funcional del apoyo social (satisfacción con el apoyo recibido), así como el cuestionario utilizado para medir el apoyo social en población sin hogar por Muñoz y Vázquez (17) para la parte estructural (frecuencia de contacto con diversas fuentes de apoyo).

Sucesos vitales estresantes (en adelante SVE): Se ha construido un instrumento ad hoc basado en la adaptación y ampliación del LTE-Q (List of Threatening Experiences Questionnaire) (18), realizado por Muñoz y Vázquez $(8,17)$ y el inventario de experiencias extremas contenido en el cuestionario VIVO (19). Para cada suceso se ha indicado el momento de ocurrencia, la afectación en la vida y su influencia en la situación sin hogar.

Salud mental y abuso de sustancias: Se ha utilizado el General Health Questionnaire (GHQ) (20), la versión al castellano de Muñoz (21). Se trata de la versión 
ORIGINALES Y REVISIONES

de 12 ítems (GHQ-12), siendo 7 de ellos negativos y 5 positivos con un formato de respuesta dicotómica (si/no). No mide específicamente psicopatología sino malestar psicológico y sufrimiento emocional. A mayor puntuación mayor malestar.

Para el abuso de sustancias se ha utilizado un cuestionario construido ad hoc basado en un sistema de indicadores de exclusión social (22). Explora la frecuencia con la que se bebe alcohol, dónde lo consume y con quién consume habitualmente. En cuanto al consumo de otras sustancias se pregunta por el tipo, la frecuencia y la vía de consumo.

Valoración del impacto vital: Se ha realizado una adaptación a la realidad de las persona sin hogar de algunas escalas cortas del Cuestionario de Valoración de Impacto Vital Ontológico (VIVO) (19). Dicho cuestionario trata de medir el impacto vital de las experiencias extremas en la identidad y el sistema de asunciones y creencias. Las escalas utilizadas son: futuro y esperanza, cambios en la identidad, cambio de prioridades, identidad de víctima, bondad del ser humano, confianza en el ser humano y aprendizaje.

Variables psicosociales: Estrategias de afrontamiento utilizadas, expectativas de futuro, percepción de control sobre su propia vida y deshumanización.

Para el análisis estadístico de los cuestionarios se ha utilizado el programa SPSS 18.0, con un intervalo de confianza del 95\% para todas las pruebas. Se han utilizado pruebas bivariadas ( $\mathrm{T}$ de Student y ANOVA), así como correlaciones de Pearson para las variables continuas y Chi-cuadrado para las variables nominales. La confirmación de la validez de los instrumentos se ha realizado a través de análisis factorial exploratorio y confirmatorio (ecuaciones estructurales) y Alfa de Cronbach.

Resultados.

\section{Variables sociodemográficas}

La muestra total de personas que han sido entrevistadas son 107. La media de edad es de 32 años, una población relativamente joven, siendo casi el 50\% de la muestra menor de 30 años. Las personas que actualmente viven en un alojamiento para personas sin hogar son algo más jóvenes que las que viven en la calle, pero sin diferencias significativas. En cuanto a los lugares de procedencia destacan las personas procedentes del Magreb seguido de las de África Subsahariana. El nivel de estudios de la mayoría son primarios, aunque hay una proporción significativa de personas que viven en un alojamiento para personas sin hogar con estudios secundarios. 
La media de tiempo residiendo en España es de 5 años y 7 meses, por lo que no estamos hablando de una población recién llegada, más del 60\% llevan más de 3 años residiendo en España. Casi la totalidad de las personas de la muestra están solteras o separadas, aunque algo menos de un tercio tiene algún familiar viviendo en España, siendo mayor la proporción en el caso de las personas que duermen actualmente en la calle.

En cuanto al estatus legal la mayoría son indocumentados, aunque destacan el $21,6 \%$ de las personas que tienen permiso de residencia y trabajo pero duermen en la calle. También es importante señalar las personas demandantes de asilo que están sin ningún apoyo social. Las experiencias con el empleo son nulas o con trabajos de pocos meses y mal remunerados

En lo referente al tiempo que se encuentran sin hogar el 66\% lleva en la calle menos de un año, siendo el 33,7\% las que llevan más de un año en la calle. El tiempo medio es de 13 meses, aunque existe una alta variabilidad con una desviación típica de 18,9 meses. No se podría considerar una población cronificada en la situación pero tampoco unos recién llegados a la calle.

\section{Sufrimiento psicológico y malestar emocional}

La escala GHQ-12 obtiene una media de 4,2 sobre una escala total de 12. Las puntuaciones son altas teniendo en cuenta que la puntuación 5 se considera como punto de corte para valorar la eventual presencia de alguna disfunción psicológica (17), y muy altas si las comparamos con la población general española (23) con una media de 2,05 .

Tabla 1:

Correlaciones de la escala GHQ con las variables sociodemográficas y de calle

\begin{tabular}{|c|c|}
\hline & GHQ-12 \\
\hline Media & 4,27 \\
\hline Desviación típica $^{\text {Edad }}$ & 2,61 \\
\hline Procedencia $^{2}$ & 0,128 \\
\hline Tiempo de estancia en España $^{1}$ & 1,263 \\
\hline Documentación $^{2}$ & $0,248^{*}$ \\
\hline Empadronamiento $^{2}$ & 1,105 \\
\hline Tiempo padrón $^{1}$ & $3,404^{*}$ \\
\hline Prestaciones económicas $^{2}$ & $-0,023$ \\
\hline Tiempo en calle & 1,240 \\
\hline Tipo de alojamiento & 0,114 \\
\hline & $4,109^{* *}$ \\
\hline
\end{tabular}

$* \mathrm{p} \leq 0,05 \quad * * \mathrm{p} \leq 0,001$

1: $\mathrm{T}$ y $\mathrm{R}$ de Pearson 2: F y $\chi^{2}$

El cuestionario GHQ ha mostrado correlación con el tiempo de residencia en España, dándose mayores puntuaciones de sufrimiento psicológico según aumenta el tiempo de residencia. Las diferencias son significativas $(\mathrm{F}=5,287 \mathrm{p} \leq 0,001)$ entre los que llevan menos de un año y los que llevan entre cinco y siete, también entre los que llevan entre uno y tres años y los que llevan entre cinco y siete años, o 
más de siete. Posiblemente, el mantenimiento de una situación social muy precaria, puesto en relación con el tiempo viviendo en el país, y las expectativas de estabilidad y progreso que se le presupone a quienes llevan mucho tiempo de residencia, influye en un mayor malestar psicológico y emocional.

También hay diferencias significativas tanto en estar empadronado (media GHQ 3,8) y no estarlo (media GHQ 5,4), como en el tipo de empadronamiento, donde se observa una tendencia a puntuaciones más altas en los empadronamientos más precarios (favores de amigos, pagar por empadronarse, etc.) frente a fórmulas más estables.

Resulta sorprendente que no se hayan encontrado diferencias en malestar psicológico y sufrimiento emocional (GHQ-12) con base en el tiempo sin hogar, incluso forzando a comparar las medias entre los que llevan menos y más de dos años sin hogar, aunque se aprecian valores medios altos en sufrimiento psicológico según aumenta el tiempo en calle. Donde sí se han encontrado diferencias significativas es en el alojamiento actual, siendo el malestar psicológico y sufrimiento emocional mayor entre quienes actualmente duermen en la calle (media 5,1) que aquellos que lo hacen en un alojamiento estable (media 3,2), independientemente del tiempo que se haya estado sin hogar, mostrando la importancia de los recursos materiales para favorecer el bienestar psicológico.

Tabla 2:

Correlaciones de la escala GHQ con otras variables

\begin{tabular}{|c|c|c|}
\hline & & GHQ-12 \\
\hline \multicolumn{2}{|c|}{ Desesperanza } & $-0,084$ \\
\hline \multicolumn{2}{|c|}{ Control de la vida } & $-0,240^{*}$ \\
\hline \multicolumn{2}{|c|}{ Deshumanización } & $0,343 * *$ \\
\hline \multirow{2}{*}{ Familia en origen } & Estructura & $-0,292 * *$ \\
\hline & Funcionalidad & 0,182 \\
\hline \multirow[b]{2}{*}{ Otras personas sin hogar } & Estructura & $-0,095$ \\
\hline & Funcionalidad & 0,188 \\
\hline \multirow{2}{*}{ Profesionales de los recursos } & Estructura & $-0,399 * *$ \\
\hline & Funcionalidad & $-0,102$ \\
\hline \multicolumn{2}{|c|}{ Consumo de alcohol } & $0,269 *$ \\
\hline \multicolumn{2}{|c|}{ Cuestionario VIVO } & $0,482 * *$ \\
\hline \multicolumn{2}{|c|}{ Cuestionario BISS } & $0,486 * *$ \\
\hline \multicolumn{2}{|c|}{ SVE vinculados a Salud mental y abuso de sustancias } & $0,214^{*}$ \\
\hline \multicolumn{2}{|c|}{ SVE Crisis y experiencias cuestionadoras } & $0,262 * *$ \\
\hline \multicolumn{2}{|c|}{ SVE Vida en la calle } & $0,213 *$ \\
\hline \multicolumn{2}{|c|}{ SVE Circunstancias vitales } & 0,114 \\
\hline \multicolumn{2}{|c|}{ SVE País de origen } & $0,410 * *$ \\
\hline
\end{tabular}

R de Pearson $\quad * \mathrm{p} \leq 0,05 \quad * * \mathrm{p} \leq 0,001$

Las personas que creen no poder controlar su vida en la actual situación puntúan significativamente más alto $(\mathrm{T}=2,534 \mathrm{p} \leq 0,05)$ en malestar psicológico (media 4,88 ) que las que consideran que sí pueden controlar su vida (media 3,64). 
De forma inversa, aquellas que consideran un grado de deshumanización mayor puntúan de manera significativa más alto $(\mathrm{T}=-3,885 \mathrm{p} \leq 0,001)$ en sufrimiento emocional $(5,42 \mathrm{Vs} 3,58)$.

La frecuencia de la relación con la familia de origen y con los profesionales de los recursos tiene una alta correlación con el malestar psicológico y el sufrimiento emocional. En este caso la correlación es negativa, lo que significa que mayor contacto con la familia de origen y profesionales de los recursos supone un menor malestar psicológico y mejor salud mental.

$\mathrm{Al}$ realizar una comparación de medias $\mathrm{T}$ para muestras independientes, sobresale el importante malestar psicológico de las personas que tienen un contacto débil o nulo con los profesionales de los recursos $(\mathrm{T}=6,768 \mathrm{p} \leq 0,05)$. La media (los que tienen contacto débil o nulo 7,30 Vs los que tienen un contacto permanente $3,95)$ señala un riesgo de psicopatología. Teniendo en cuenta que un alto porcentaje de la muestra mantiene contacto con los profesionales de los recursos, estaríamos hablando de un reducido grupo de personas cuya desafiliación les haya hecho cortar el principal lazo de unión que mantienen la mayoría de las personas sin hogar entrevistadas.

Las personas que tienen un contacto débil o nulo con la familia de origen obtienen una puntuación mayor de malestar psicológico (media 5,12 ) que las que mantienen un contacto permanente (media 3,77), aunque no tan acusado como las diferencias con los profesionales de los recursos $(T=2,72 p \leq 0,05)$.

No se encuentra relación entre la satisfacción media con el apoyo social y el malestar psicológico, aunque las comparaciones de medias muestran puntuaciones significativamente más altas en el cuestionario GHQ en aquellas personas que consideran satisfactorio el apoyo de otras personas $\sin \operatorname{hogar}(\mathrm{T}=2,599 \mathrm{p} \leq 0,05)$ y lo contrario con los profesionales de los recursos $(\mathrm{T}=2,188 \mathrm{p} \leq 0,05)$.

Las personas que en la actualidad hacen un uso frecuente del alcohol (beben tres o más días a la semana) obtienen puntuaciones medias significativamente más altas en la escala GHQ $(T=-3,389 \mathrm{p} \leq 0,05)$ que aquellas con un consumo moderado $(6,07$ Vs 4,00$)$

Por último, es destacable la alta correlación entre las puntuaciones del GHQ y de los cuestionarios VIVO y BISS, tanto en la puntuación total como con cada una de las subescalas. Guarda una lógica el que a mayor estrés aculturativo se dé, a su vez, un mayor malestar psicológico o sufrimiento emocional, y que un mayor impacto vital, también suponga un incremento de malestar psicológico. En referencia a las sub-escalas, es significativo que la mayor correlación del GHQ-12 se dé con las dos sub-escalas del BISS que remiten al contexto social, las condiciones que rodean a la persona o la situación social en el país de "acogida", esto es, la sub-escala de discriminación percibida y la sub-escala de estrés psicosocial general (claramente vinculado a los aspectos materiales de la vida de la persona). En rela- 
ORIGINALES Y REVISIONES

ción a las sub-escalas del VIVO la mayor correlación se da con la identidad, esto es, un mayor impacto identitario también supone un mayor sufrimiento emocional.

\section{Otras medidas.}

Se deja para el final las correlaciones del GHQ con los sucesos vitales estresantes ya que, algunos de ellos, nos ayudan a comprender el sufrimiento psicológico de las personas migrantes sin hogar y las problemáticas de salud mental anteriores. Destacan los siguientes datos:

Tabla 3:

Sucesos vitales estresantes padecidos a lo largo de la vida

\begin{tabular}{|c|c|c|c|c|c|}
\hline & Ocurrencia $^{1}$ & $\begin{array}{c}\text { Antes de estar sin } \\
\text { hogar }^{1}\end{array}$ & $\begin{array}{l}\text { Después de estar } \\
\text { sin } \text { hogar }^{1}\end{array}$ & $\begin{array}{c}\text { Afectación en la } \\
\text { vida }^{2}\end{array}$ & $\begin{array}{c}\text { Influencia en el } \\
\text { sinhogarismo }^{2}\end{array}$ \\
\hline $\begin{array}{l}\text { Beber alcohol en } \\
\text { exceso en alguna } \\
\text { época }(n=49)\end{array}$ & $45,8 \%$ & $24,3 \%$ & $35,5 \%$ & $83,7 \%$ & $65,3 \%$ \\
\hline $\begin{array}{l}\text { Usar drogas en } \\
\text { exceso en alguna } \\
\text { época }(n=29)\end{array}$ & $27,1 \%$ & $22,4 \%$ & $16,8 \%$ & $75,9 \%$ & $48,3 \%$ \\
\hline $\begin{array}{l}\text { Haber estado } \\
\text { ingresado en } \\
\text { un hospital } \\
\text { psiquiátrico }(n=7)\end{array}$ & $6,5 \%$ & $3,7 \%$ & $2,8 \%$ & $85,7 \%$ & $57,1 \%$ \\
\hline $\begin{array}{l}\text { Padecer algún } \\
\text { problema de salud } \\
\text { mental grave }(n=8)\end{array}$ & $7,5 \%$ & $4,7 \%$ & $5,6 \%$ & $100 \%$ & $75 \%$ \\
\hline $\begin{array}{l}\text { Ha intentado } \\
\text { suicidarse }(n=14)\end{array}$ & $13,1 \%$ & $4,7 \%$ & $9,3 \%$ & $100 \%$ & $35,7 \%$ \\
\hline
\end{tabular}

El porcentaje de personas que refieren algún problema de salud mental grave en su vida es menor que los datos aportados por algunos estudios epidemiológicos que presentan una prevalencia vida del $19,5 \%$ para los trastornos mentales en población general (24). La diferencia puede ser debida a múltiples razones como la diferencia de los instrumentos utilizados (en la presente investigación no se ha utilizado ningún instrumento estandarizado, tan solo una pregunta sobre la presencia de enfermedad), la falta de conciencia de enfermedad por parte de las personas entrevistadas, la edad media de la muestra y también puede ser reflejo de las dificultades de acceso de esta población a los servicios de salud mental, invisibilizando la problemática.

En España la prevalencia de ideas suicidas es el de 4,4\% y los intentos del 1,5\% (25). Los entrevistados informan en un $13,1 \%$ de intentos de suicidio, triplicando el porcentaje de población general. El dato concuerda con los relatos de la dureza de la 
vida en la calle, con las puntuaciones obtenidas en la escala de malestar psicológico y sufrimiento emocional y con la importante ocurrencia de sucesos vitales estresantes.

Tabla 4:

Comparación de medias de la escala GHQ-12 con base en sucesos vitales estresantes.

\begin{tabular}{|c|c|c|c|c|c|}
\hline & & Media GHQ & $\begin{array}{c}\text { Desviación } \\
\text { típica }\end{array}$ & $\mathbf{N}$ & $\mathbf{F}$ \\
\hline \multirow{6}{*}{$\begin{array}{l}\text { Salud mental } \\
\text { y abuso de } \\
\text { sustancias }\end{array}$} & 0 Sucesos & 3,57 & 2,50 & 47 & \multirow{6}{*}{1,474} \\
\hline & 1 Suceso & 4,72 & 2,27 & 32 & \\
\hline & 2 Sucesos & 4,68 & 3,21 & 16 & \\
\hline & 3 Sucesos & 5,50 & 1,37 & 6 & \\
\hline & 4 Sucesos & 4,60 & 3,78 & 5 & \\
\hline & 5 Sucesos & 7,00 & - & 1 & \\
\hline \multirow{5}{*}{$\begin{array}{c}\text { Crisis y } \\
\text { experiencias } \\
\text { cuestionadoras }\end{array}$} & 0 Sucesos & 2,70 & 2,61 & 17 & \multirow{5}{*}{$3,478 *$} \\
\hline & 1 Suceso & 3,97 & 2,44 & 35 & \\
\hline & 2 Sucesos & 5,13 & 2,49 & 44 & \\
\hline & 3 Sucesos & 3,90 & 2,46 & 10 & \\
\hline & 4 Sucesos & 7,00 & - & 1 & \\
\hline \multirow{4}{*}{$\begin{array}{c}\text { Hechos } \\
\text { vinculados a la } \\
\text { vida en calle }\end{array}$} & 0 Sucesos & 3,47 & 2,29 & 17 & \multirow{4}{*}{2,658} \\
\hline & 1 Suceso & 3,56 & 2,55 & 32 & \\
\hline & 2 Sucesos & 5,05 & 2,43 & 34 & \\
\hline & 3 Sucesos & 4,66 & 2,85 & 24 & \\
\hline \multirow{4}{*}{$\begin{array}{l}\text { País de origen } \\
\text { y proceso } \\
\text { migratorio }\end{array}$} & 0 Sucesos & 3,30 & 2,47 & 56 & \multirow{4}{*}{$7,219 * *$} \\
\hline & 1 Suceso & 4,90 & 2,50 & 39 & \\
\hline & 2 Sucesos & 6,30 & 1,50 & 9 & \\
\hline & 3 Sucesos & 6,60 & 0,57 & 3 & \\
\hline
\end{tabular}

$* \mathrm{p} \leq 0,05 \quad * * \mathrm{p} \leq 0,01$

Una mayor ocurrencia de los sucesos vitales estresantes vinculados a la salud mental y al abuso de sustancias (beber en exceso en alguna época de la vida, usar drogas en exceso en alguna época, haber estado ingresado en un hospital psiquiátrico, padecer algún problema de salud mental grave y haber intentado suicidarse) conlleva un mayor malestar psicológico y sufrimiento emocional. Dicha relación también se da en los sucesos vinculados a la vida en la calle (sufrir amenazas de muerte, agresiones o palizas, haber sido víctima de un asalto o robo y sufrir una agresión o insulto racista), así como en los denominados "crisis y experiencias cuestionadoras" (divorcio/ruptura de relación afectiva estable, padecer problemas graves relacionados con el empleo, estar en prisión y haber estado en algún centro de menores). Para los hechos vinculados al país de origen y la migración (catástrofe natural con pérdidas personales importantes, haber sufrido situaciones de amenaza para su integridad en el país de origen y durante el viaje migratorio) se da la paradoja de una destacada correlación entre su ocurrencia y el malestar psicológico, a pesar de que la escala evalúa cómo se ha sentido la persona en las últimas semanas y los sucesos refieren a experiencias ocurridas antes de la migración y por lo tanto, en la mayoría de los casos lejanas en el tiempo. 
ORIGINALES Y REVISIONES

\section{Conclusiones.}

Apenas existe investigación en cuestiones relacionadas con salud mental y exclusión social y aquellas que se han dado se han centrado en diagnósticos, tasas de prevalencia, etc. Se trata, indudablemente, de investigaciones esenciales que permiten acotar la problemática. Sin embrago, el excesivo énfasis en este tipo de investigaciones conlleva dos problemáticas importantes. La primera es que ahondan en la estigmatización de colectivos ya de por si altamente estigmatizados como las personas sin hogar o las personas migrantes. Es la visión de los excluidos como "underclass" (26-27) caracterizado por un avanzado estado de degradación moral y personal que impide a sus miembros asumir el trabajo regular y legal como forma de vida y la familia como institución de reproducción social. Por otra parte, se descontextualiza el problema centrando la atención en las características de las personas y no en el componente estructural o social de la exclusión. Cualquier investigación sobre la exclusión social debería combinar los factores individuales y de experiencias sufridas por la persona (micro procesos) con factores estructurales (macro procesos).

La investigación presentada pretende dar pasos en este sentido y muestra una importante relación entre los recursos materiales (en muchos casos producto de factores estructurales y política sociales) y el bienestar psicológico. Así, variables como la posesión de un empadronamiento, el tipo de empadronamiento conseguido y el alojamiento en el que se reside actualmente muestra correlaciones importantes con el malestar psicológico y el sufrimiento emocional. A ello se une la importancia de otras variables como el apoyo social, en este caso concreto el mayor contacto con la familia de origen y con los profesionales de los recursos, como proveedores de un apoyo con influencia directa en el bienestar de la persona a pesar de que, en muchos casos, no puede dar un apoyo material.

La investigación evidencia un 48,6\% de las personas entrevistadas que obtienen 5 o más puntos en el cuestionario, considerando el 5 como punto de corte para valorar la eventual presencia de alguna disfunción psicológica. En la investigación realizada en Madrid con personas sin hogar (17) casi el 70\% de las personas entrevistadas superaba dicha puntuación, reflejando un mayor malestar psicológico que las personas entrevistadas para la presente investigación. Las diferencias en las muestras de ambas investigaciones pueden explicar los resultados encontrados, al darse mayores problemáticas personales en las personas nacionales sin hogar. Ejemplo de ello es el menor padecimiento de sucesos vitales estresantes. Pese a ello se comprueba el padecimiento de un importante número de sucesos vitales estresantes, en algunos casos con importante afectación en la vida de la persona como los intentos de suicidio o los ingresos en psiquiatría. La pregunta que debemos hacernos es ¿cómo es posible encontrarnos con personas que han pasado por 
ingresos psiquiátricos durmiendo en la calle? La falta de desarrollo de recursos sociosanitarios que permitan dar una respuesta adecuada a este tipo de población es una de las respuestas válidas para dicha pregunta.

El desarrollo de los mismos en vital en vista de la importancia que ha mostrado la investigación en los recursos materiales como variable influyente en el bienestar psicológico. Otras variables influyentes han sido la sensación de deshumanización, la falta de control sobre la propia vida, el consumo de alcohol o los cambios identitarios producidos por la vida en la calle. Poner en relación estas variables a través de estudios multivariantes nos dará una visión mucho más realista de los problemas de salud mental de las personas que sufren exclusión social y nos permitirá realizar intervenciones situadas, y por lo tanto más adecuadas a la problemática sobre la que se interviene.

\section{BIBLIOGRAFÍA}

(1) Goodman, L., Saxe, L., \& Harvey, M. Homelessness as psychological trauma. Broadening perspectives. American Psychologist, 46(11), (1991). 1219-1225.

(2) Thornicroft, G., \& Tansella, M. The mental Health Matrix. A manual to improve services. Cambridge: Cambridge University Press. (1999)

(3) Robertson, M. J. The prevalence of mental disorders among homeless people. In R. I. Jahiel (Ed.), Homelessness. New York: John Hopkins Univsersity Press. (1992).

(4) Fazel, S., Khosla, V., Doll, H., \& Geddes, J. The prevalence of mental disorders among the homeless in western countries: systematic review and meta-regression analysis. PLoS medicine, (2008). 5(12).

(5) Cabrera, P., \& Rubio, M. J. Las personas sin hogar, hoy. Revista del Ministerio de Trabajo e Inmigración, (2008).75, 51-74.

(6) Cabrera, P. Huéspedes Del Aire: Sociología de Las Personas Sin Hogar en Madrid. Madrid: Universidad Pontificia de Comillas. (1998).

(7) Lucas, R., Batista, G., Borrás, V., Catarineau, S., Sánchez-Sahis, I., \& Valls, E. Prevalencia de sintomatología psicótica y hábitos tóxicos en una muestra de "homeless". Revista de Psiquiatría de la Facultad de Medicina de Barcelona, (1995). 22(1), 18-24.

(8) Muñoz, M., Vázquez, C., \& Cruzado, J. A. Personas sin hogar en Madrid: Informe psicosocial y epidemiológico. Madrid: Comunidad de Madrid. (1995).

(9) Muñoz, M., Vázquez, J. J., Panadero, S., \& Vázquez, C. Características de las personas sin hogar en España: 30 años de estudios empíricos. Cuadernos de Psiquiatría Comunitaria, (2003). $3(2), 100-116$.

(10) Gómez, M. Estrategia en salud mental del sistema nacional de salud, 2006. Madrid: Ministerio de Sanidad y Consumo. Madrid. (2007).

(11) Swinnen, S. G. H. A., \& Selten, J.-P. Mood disorders and migration: meta-analysis. The British Journal of Psychiatry, (2007). 190, 6-10. 
(12) Achotegui, J. La depresión en los inmigrantes: una perspectiva transcultural. Barcelona: Mayo. (2002).

(13) Achotegui, J. Estrés límite y salud mental: el síndrome del inmigrante con estrés crónico y múltiple (síndrome de Ulises). Migraciones, (2006). (19), 59-85.

(14) Tomás-Sábado, J., Qureshi, A., Antonin, M., \& Collazos, F. Construction and preliminary validation of the Barcelona Immigration Stress Scale. Psychological reports, (2007). 100(3 Pt 1), 1013-23.

(15) Martínez, M., García, M., \& Maya, I. Pretest del cuestionario de evaluación de necesidades y recursos, y del ASSIS de Barrera. Sevilla. (1993)

(16) Barrera, M. A method for the assessment of social support networks in community survey research. Connections, (1980). (3), 8-13.

(17) Muñoz, M., Vázquez, C., \& Vázquez, J. J. Los límites de la exclusión: Estudio sobre los factores económicos psicosociales y de salud que afectan a las personas sin hogar en Madrid. Madrid: Ediciones Témpora. (2003).

(18) Brugha, T. S., \& Cragg, D. The List of Threatening Experiences: the reliability and validity of a brief life events questionnaire. Acta Psychiatrica Scandinavica, (1990). 82(1), 77-81.

(19) Pérez-Sales, P., Eiroa-Orosa, F. J., Olivos, P., Barbero-Val, E., Fernández-Liria, A., \& Vergara, M. Vivo Questionnaire: A Measure of Human Worldviews and Identity in Trauma, Crisis, and Loss - Validation and Preliminary Findings. Journal of Loss and Trauma, (2012). 17(3), 236-259.

(20) Goldberg, D. P., \& Williams, P. A user's guide to the General Health Questionnaire. Windsor, Berks: NFER-Nelson. (1988).

(21) Lobo, A., Muñoz, P., Goldberg, D., \& Williams, P. Cuestionario de salud general GHQ (General Health Questionnaire). Versiones en lengua española validadas. GHQ (General Health Questionnaire). Barcelona: Masson. (2010).

(22) Vidal, F., Mota, R., Lázaro, S., Rubio, E., \& Iglesias, J. Ensayo de sistema de indicadores sobre exclusión social. La exclusión social y el estado de bienestar en España (pp. 741-806). Barcelona: Icaria. (2006).

(23) Rocha, K., Pérez, K., Rodríguez-Sanz, M., Borrell, C., \& Obiols, J. E. Propiedades psicométricas y valores normativos del General Health Questionnaire (GHQ-12) en población general española. International Journal of Clinical and Health Psychology, (2011). 11(1), 125-139.

(24) Haro, J. M., Palacín, C., Vilagut, G., Martínez, M., Bernal, M., Luque, I., Codony, M., et al. Prevalencia de los trastornos mentales y factores asociados: resultados del estudio ESEMeDEspaña. Medicina Clínica, (2006). 126(12), 445-451.

(25) Gabilondo, A., Alonso, J., Pinto-Meza, A., Vilagut, G., Fernández, A., Serrano-Blanco, A., Almansa, J., et al. Prevalencia y factores de riesgo de las ideas, planes e intentos de suicidio en la población general española. Resultados del estudio ESEMeD. Medicina Clínica, (2007). 129(13), 494-500.

(26) Myrdal, G. Challenge to affluence. New York: Phanteon. (1962).

(27) Wilson, W. J. The Truly Disadvantaged: The Inner City, the Underclass, and Public Policy. (G. Bridge \& S. Watson, Eds.) Chicago London (Vol. 111, p. 254). University of Chicago Press. (1987). 